\title{
Study of bloodstream of the white rats stomach and small intestine
}

\author{
Authors: Daria Maryniak ${ }^{1}$, Volodymyr $\operatorname{Hryn}^{2}$ (mentor) \\ ${ }^{1}$ Department of Human Anatomy, Ukrainian Medical Stomatological Academy, Poltava, Ukraine
}

DOI: https://doi.org/10.26800/LV-142-supp5-41

Introduction:

The information on the anatomical and physiological features of the gastrointestinal tract of white rats are reported within publications of many authors who are engaged in experimental modelling of the pathological states of the digestive system.

Aim:

The paper was geared toward the study and systematization of the features of the angioarchitecture of the white rat's stomach and tiny intestine.

\section{Materials and methods:}

The study based on the injection of the blood vasculature of abdominal organs of 20 white male rats with $5 \%$ gelatine solution, coloured with filtered black ink, was performed in the mode of maintaining the temperature of solution within the range of $37-40^{\circ} \mathrm{C}$. Euthanasia of rats were made under thiopentone anaesthesia overdose $(75 \mathrm{mg} / \mathrm{kg}$ animal body weight intramuscularly in the upper third of the hip). Then the animal's corpse was embedded first into cold water and fixed in a $10 \%$ formalin solution for 2 days. The whole complex of internal organs was removed from the abdominal cavity of the animal and partitioned into sections. Photographing made by a digital camera, binocular magnifier MBS-9, equipped with Sigeta DCM-900 9.0MP.

\section{Results:}

In the stomach, the highest concentration of blood microvessels is in the glandular part. In the small intestine, the typical principle of the microvasculature organization of its mucous membrane is modified within the duodenum, which is related to the presence of Brunner's glands and group of Peyer's patches.

\section{Conclusion:}

The results of injecting blood vasculature of white rats' gastrointestinal tract with ink mass clearly demonstrate the specific difference within the intraorganic angioarchitecture of its different regions.

Keywords: bloodstream, mucous membrane, small intestine, stomach. 